\title{
Brainstem hemorrhage after neural therapy for decreased libido in a 31-year-old woman
}

\author{
Christian A. Schmittinger • Ralph Schär • \\ Christian Fung • Werner J. Z'Graggen • \\ Claude Nauer · Martin W. Dünser $\cdot$ Simon Jung
}

Received: 17 December 2010/ Accepted: 17 January 2011/Published online: 1 February 2011

(C) Springer-Verlag 2011

\section{Dear Sirs,}

In September 2010, a 31-year-old otherwise healthy, female Caucasian consulted a dermatologist with additional qualifications in neural therapy because of decreased libido resistant to treatment. Following unproblematic procaine $1 \%$ injections at multiple gynecological and thyroidal sites, per os epipharyngeal injection was performed intending to infiltrate a neural therapy trigger point close to the pharyngeal tonsil at the anterior aspect of the sphenoid bone. This target point is assumed to represent remnants of the craniopharyngeal duct and Rathke's pouch and is considered the source of various hormone imbalances [1]. Immediately after injection of $1 \mathrm{ml}$ procaine, the patient developed right-sided brachiocrural hemiparesis, numbness of all limbs, nausea, vomiting, and rotatory vertigo. Approximately $30 \mathrm{~min}$ later, sensorimotor deficits gradually subsided, the vertiginous patient was reassured and sent home with an appointment set for the next day. Since the patient still complained about vertigo, nausea, and repeated vomitus on the following day, she was admitted to

C. A. Schmittinger - M. W. Dünser

Department of Intensive Care Medicine, Inselspital,

Bern Medical University, Bern, Switzerland

R. Schär · C. Fung · W. J. Z'Graggen

Department of Neurosurgery, Inselspital,

Bern Medical University, Bern, Switzerland

C. Nauer

Institute for Diagnostic and Interventional Neuroradiology,

Inselspital, Bern Medical University, Bern, Switzerland

\section{S. Jung $(\bowtie)$}

Department of Neurology, Inselspital, Bern Medical University,

Freiburgstrasse 18, 3010 Bern, Switzerland

e-mail: simon.jung@insel.ch the emergency department of our hospital. At initial presentation, the patient was fully alert and orientated. The clinical examination was notable for positional vertigo in right lateral position and gaze-provoked upbeat nystagmus. Furthermore, a slight deficit in sensibility on the anterior aspect of the right lower leg was detected. The remainder of the clinical examination and all laboratory results were normal. Magnetic resonance imaging revealed a hemorrhagic lesion of $3 \mathrm{~mm}$ in diameter in the left paramedian medulla oblongata with slight perifocal edema (Fig. 1). Contrast-enhanced magnetic resonance angiography showed no evidence of any vertebral or intracranial artery dissection. Hereafter the patient was transferred to the intermediate care unit for further observation and was started on ceftriaxone and metronidazole to cover for possible contamination of the cerebrospinal fluid and brain by enoral microflora. She made an uneventful recovery, which allowed transfer to the neurology ward on the following day. Six days after admission, the patient was discharged from the hospital without a neurological deficit or subjective discomfort. To what extent the primordial desire was influenced by this experience was not ascertainable.

Neural therapy was first described by Walter and Ferdinand Huneke in 1925 [1]. Although lacking scientific evidence, it is a widely used complementary medical method in Europe to treat acute and chronic pain syndromes, circulatory, autoimmune, and vegetative dysregulations [1]. Neural therapy uses injections of local anesthetics into or close to pathologically altered body regions such as scars, peripheral nerves, autonomic ganglia, glands, or other trigger points. Numerous adverse events have been reported following neural therapy, particularly when deep structures or internal organs were targeted [2-4]. So far, only one case of central nervous system hemorrhage has been reported. In 1979, Heyll and 
Fig. 1 Magnetic resonance imaging. a Axial susceptibility weighted image showing the small hemorrhage as a hypointense dot in the medulla oblongata (arrow). b T2weighted axial view showing the intramedullar hemorrhage (white dotted arrow) with surrounding edema. c Sagittal T1 weighted image showing intramedullar hemorrhage (short white arrow), the supposed needle direction (long white arrow), and the originally desired target point (white asterisk)
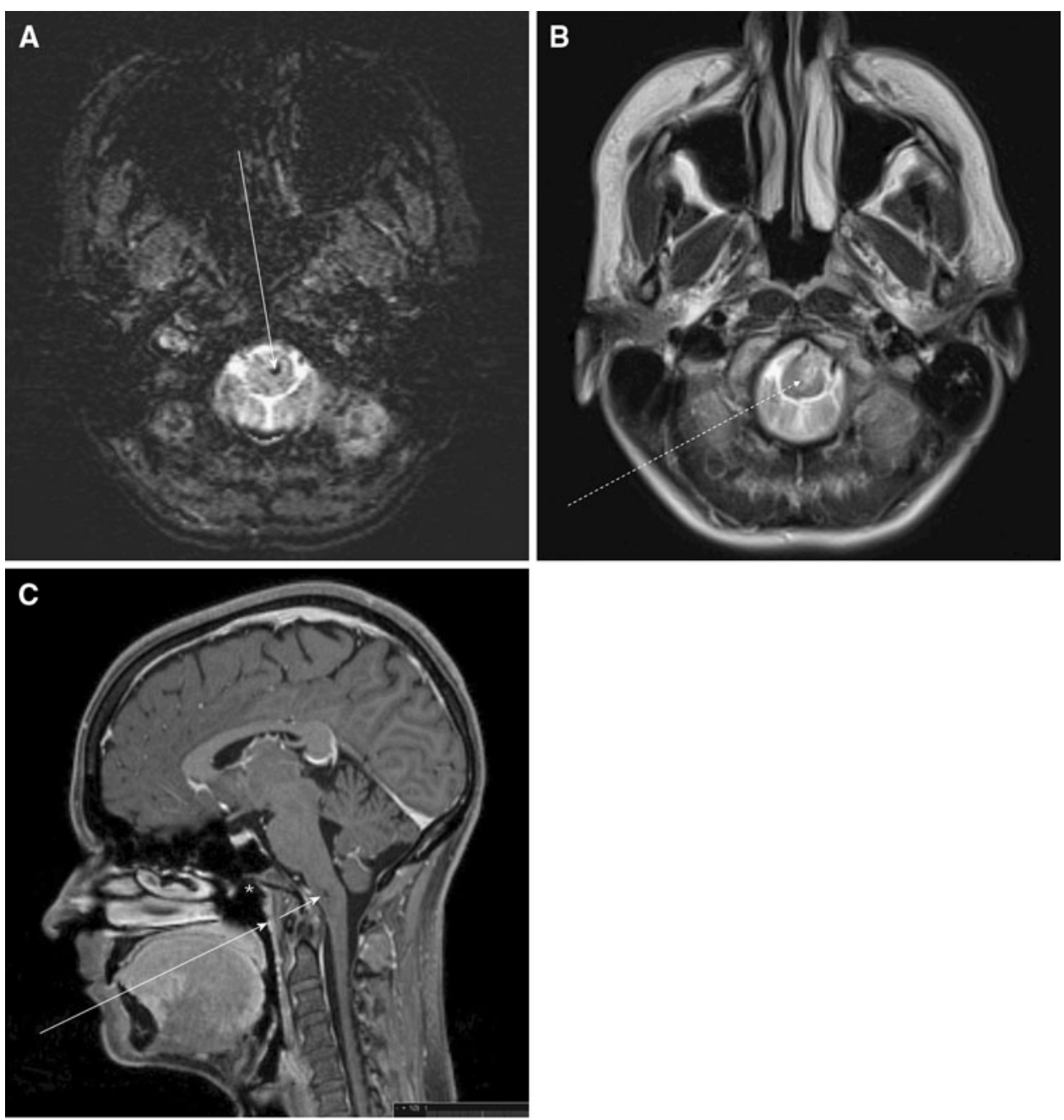

Ziegenhagen described a 37-year-old female patient who developed subarachnoid hemorrhage after infiltration of the pharyngeal tonsil [4]. In our case, the needle used by the neural therapist missed the intended target since the actual trajectory was too caudal passing between the basilar part of the occipital bone and the odontoid process, resulting in puncture of the brain stem and causing intramedullary hemorrhage. Transient sensorimotor signs were potentially precipitated by the injected local anesthetic. In conclusion, this was a fortunate course of an iatrogenic brainstem injury but this must give cause for serious concern that invasive complementary medical methods are not entirely safe and may be associated with serious, potentially lifethreatening side-effects, similar to those observed in conventional medicine. The number of reports on adverse effects of neural therapy is still low but respectability suggests that neural therapy should only be performed by trained physicians aware of the possible risks and complications of this technique. Furthermore, patients undergoing such therapy must be advised of its potential hazards. The patient's written consent was obtained and permission to publish was given.

Conflict of interest No author has a conflict of interest in regards to the drugs or methods discussed in this manuscript.

\section{References}

1. Fischer L (2001) Neuraltherapie nach Huneke. Hippokrates-Verlag $\mathrm{GmbH}$, Stuttgart, pp 123-124

2. Mattig W, Buchholz W, Schulz HJ (1979) Severe iatrogenic lesions caused by Huneke's neural therapy. Z Gesamte Inn Med 34:143-147

3. Stohr M, Mayer K (1976) Nerve-root damage from local injections. Dtsch Med Wochenschr 101:1218-1220

4. Heyll U, Ziegenhagen DJ (2000) Subarachnoid hemorrhage as lifethreatening complication of neural therapy. Versicherungsmedizin $52: 33-36$ 